\title{
Diseño de un sistema de realidad aumentada para la prevención de obesidad en niños
}

\author{
Oseas Neftali Gómez Soto ${ }^{1}$, Mabel Vazquez Briseño ${ }^{1}$, \\ Mónica Elizabeth Tentori Espinosa ${ }^{2}$ \\ ${ }^{1}$ Universidad Autónoma de Baja California, Maestría y Doctorado en Ciencias e Ingeniería, \\ México \\ ${ }^{2}$ Centro de Investigación Científica y de Educación Superior de Ensenada, Departamento de \\ Ciencias de la Computación, México \\ \{neftali, mabel.vazquez\}@uabc.edu.mx, mtentori@cicese.mx
}

\begin{abstract}
Resumen. México tiene una prevalencia significativa en sobrepeso y obesidad infantil, uno de los factores que influye es el consumo desbalanceado de alimentos. Muchos de los alimentos procesados actualmente incluyen etiquetas mostrando su información nutrimental, el problema radica en que los niños antes de consumir los alimentos no leen estas etiquetas, no las entienden y no analizan si deben consumir ese alimento. Como una solución a este problema, en este trabajo se presenta el diseño de un sistema interactivo que permita mostrar información nutrimental a niños de manera clara utilizando Realidad Aumentada (RA). El diseño se realizó a través de entrevistas y sesiones de diseño participativo, logrando implementar un prototipo de baja fidelidad para una aplicación móvil orientada a la prevención de obesidad. Además se realizó una evaluación de frameworks de RA con el fin de conocer cuál es el apropiado para el desarrollo del sistema.
\end{abstract}

Palabras clave: Realidad aumentada, dispositivos móviles, rapid contextual design, sistema interactivo, obesidad infantil, framework.

\section{Introducción}

La obesidad infantil es un problema mundial que afecta significativamente a México. De acuerdo con datos del Fondo de las Naciones Unidas para la Infancia (UNICEF, por sus siglas en inglés) [1] México ocupa el primer lugar mundial en obesidad infantil y segundo lugar en obesidad en adultos. Según la Encuesta Nacional de Salud y Nutrición (ENSANUT) [2] México tiene una prevalencia de sobrepeso y obesidad en niños de 5-11 años de edad en 2012 de $36.9 \%$ (17.4 en obesidad y 19.5 en sobrepeso).

Una de las causas de obesidad de acuerdo con la Organización Mundial de la Salud (OMS) [3] es el aumento de la ingesta de alimentos hipercalóricos con abundantes grasas y azúcares, pero con escasas vitaminas, minerales y otros micronutrientes saludables. El consumo excesivo de alimentos hipercalóricos en ocasiones se debe al desconocimiento o poco entendimiento de los nutrimentos que contiene cada alimento. 
Considerando esta problemática y las herramientas tecnológicas disponibles, es conveniente apoyar a la prevención de la obesidad infantil con el desarrollo de nuevas herramientas que colaboren para tener una mejor alimentación. Una de las maneras es por medio de un sistema de salud móvil ( $m$-Health) orientado a niños que de manera atractiva y amigable les permita interactuar con su Smartphone o Tablet, brindándoles así información acerca de los alimentos utilizando RA. Desde la perspectiva de Billinghurst [4] la RA permite que incluso los niños muy pequeños puedan tener una experiencia educativa rica por medio de ésta.

Para desarrollar proyectos de RA, existen algunos componentes fundamentales, como son: cámaras, marcadores, pantallas, información virtual y software especializado en RA (ver Fig. 1).

A partir del avance tecnológico que presentan hoy en día los dispositivos de comunicación móvil, como son los Smartphones, y considerando los requerimientos antes mencionados, la RA se puede desarrollar utilizando un dispositivo de este tipo, ya que cuenta con estas capacidades y con las herramientas para desarrollarla.

Por otra parte al desarrollar un sistema es de importancia que este tenga buena usabilidad y experiencia de usuario [6]. Considerando este factor, así como el problema social y las ventajas de la RA, se diseñó un sistema interactivo que utiliza RA, enfocado en fomentar una correcta alimentación de los niños de seis a ocho años.

El presente trabajo muestra un diseño interactivo como resultado obtenido por medio de Rapid Contextual Design bajo la filosofía de diseño centrado en el usuario. Por otra parte muestra algunas pruebas que se hicieron a frameworks de RA dando como conclusión una opción apropiada para el futuro desarrollo del sistema.

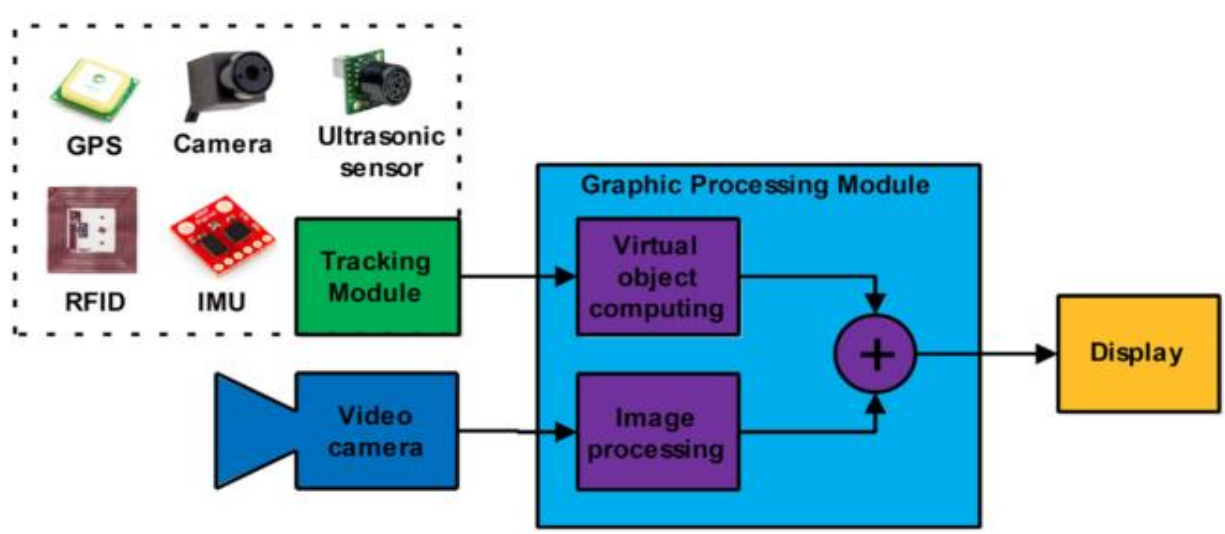

Fig. 1. Arquitectura de RA [5].

\section{Trabajo Relacionado}

De acuerdo con Santos [7], la tecnología se puede utilizar para cumplir con diferentes funciones en el área de educación, una de éstas es enriquecer e incrementar la eficiencia de la presentación de contenidos, ya que la tecnología tiene affordances que cambian la experiencia de aprendizaje. 
Desde la perspectiva de Bayu et al [8], la visualización de información con RA es un método muy eficaz para la expansión del proceso de información visual, sobre todo en el área relacionada con la salud y el uso de dispositivos móviles. Una de las aplicaciones que se han desarrollado en dicha área es Vitals Signs Camera, desarrollada por Philips [9], ésta mide el ritmo cardíaco y respiratorio por medio del iPad o iPhone. Por otra parte Bayu et al [8] desarrollaron una aplicación en la plataforma Android que proporciona información nutrimental utilizando un dispositivo móvil, que mediante el uso de la cámara identifica alimentos y les añade información nutrimental.

Considerando las ventajas que ofrece la RA a través de los dispositivos móviles es pertinente pensar que sería de utilidad desarrollar un sistema que además de tomar ventaja de esos atributos se le aplique un diseño interactivo y dirigido a niños.

\section{Diseño del sistema}

Desde la perspectiva de Billinghurst [4], la RA permite que incluso los niños muy pequeños puedan tener una experiencia educativa rica, sin embargo antes de empezar a desarrollar una aplicación es importante conocer y entender las necesidades de los usuarios, sus prácticas actuales y prácticas ideales.

En este trabajo, para entender el contexto de los usuarios involucrados se hicieron entrevistas y sesiones de diseño. Se identificaron cuatro tipos de personas que están relacionados con la problemática y por ende con el diseño del sistema, estas son niños, padres, pediatra experto en nutrición y profesor de educación básica, con quienes se realizaron tres sesiones de diseño y cuatro entrevistas (una entrevista por cada tipo de persona). Las entrevistas realizadas constaron de preguntas relacionadas con hábitos alimenticios, conocimientos sobre alimentación, educación alimenticia, motivación a una buena alimentación y sugerencias.

Con base en las entrevistas realizadas, se elaboró un diagrama de afinidad. Este diagrama contiene ideas, temas, escenarios de uso, etc, obtenidos de las entrevistas realizadas.

Siguiendo Rapid Contextual Design se realizaron tres sesiones de diseño participativo. En la primera sesión de diseño participativo se realizó visioning, esta sesión fue materializada a través de sketches, se realizó una lluvia de ideas, las cuales se dibujaron hasta obtener los sketches (ver Fig. 2). En la segunda sesión se hizo storyboarding, primeramente se dieron a conocer las fortalezas y debilidades de cada sketch, posteriormente se eligió el sketch en el que se trabajaría en la siguiente sesión (se eligió "evidenciando el valor nutrimental"), de éste se definió como sería la interacción general entre el usuario y el sistema. En la tercera sesión se utilizó el sketch obtenido en la sesión anterior y sobre éste se hizo el prototipo, en esta sesión además se detalló cómo sería la interacción del usuario con el sistema, se definieron las pantallas del sistema y su diseño. Como resultado se obtuvo un prototipo de baja fidelidad.

A partir del proceso anterior y del análisis de datos, se definió el escenario de uso y los componentes necesarios en cuanto a software y hardware. El escenario bajo el cual se utilizaría estaría dado cuando el usuario (niño) tiene la intención de consumir un alimento y desea saber que tan saludable es dicho alimento. Así el usuario podrá, con el dispositivo móvil, conocer el contenido nutrimental de dicho alimento, dándole a 
conocer de manera entendible el valor nutrimental. Este sistema tratará de hacerle ver al usuario qué tan saludable es ese alimento, con la intención de que reflexione si lo consume o no. De esta forma, el sistema le dará puntos por registrar los alimentos obtenidos, independientemente si son saludables o no (mayor puntos por alimentos saludables). Ya que el objetivo es que registre los alimentos que consume, este registro lo podrá ver el padre en su dispositivo móvil con el fin de que conozca los alimentos consumidos por el niño para recompensarlo o alentarlo a una alimentación adecuada. Para dar a conocer el valor nutrimental al niño se utilizará RA, usando la cámara del dispositivo móvil. Al enfocar con la cámara a un alimento, esta información nutrimental se mostrará. En la Fig. 3 se muestra la estructura e interacción de los componentes del sistema a grandes rasgos.

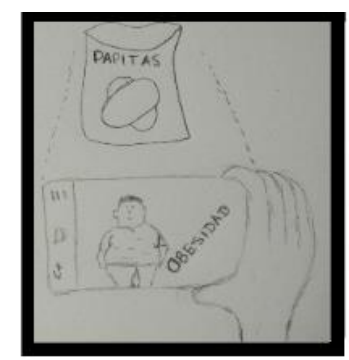

a) DISTORSIÓN DE LA REALIDAD

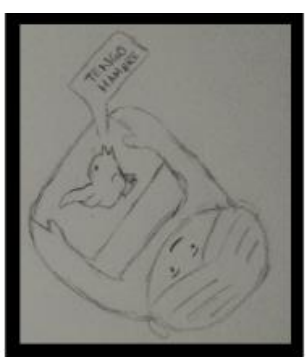

b) Tamagotchi

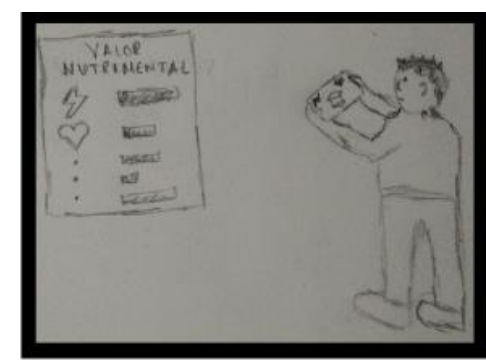

c) Evidenciando el valor nutrimental

Fig. 2. Sketches.

Como se puede observar en la Fig. 2, la primera opción tiene como finalidad representar el daño en la salud que pueda ocasionar el consumo excesivo de ese alimento. El objetivo de la segunda opción es realizar un tamagotchi que cambiará su aspecto físico dependiendo de los alimentos que consuma. La tercera opción tiene como fin mostrar de manera entendible para el niño la información nutrimental de alimentos conocidos como "chatarra".

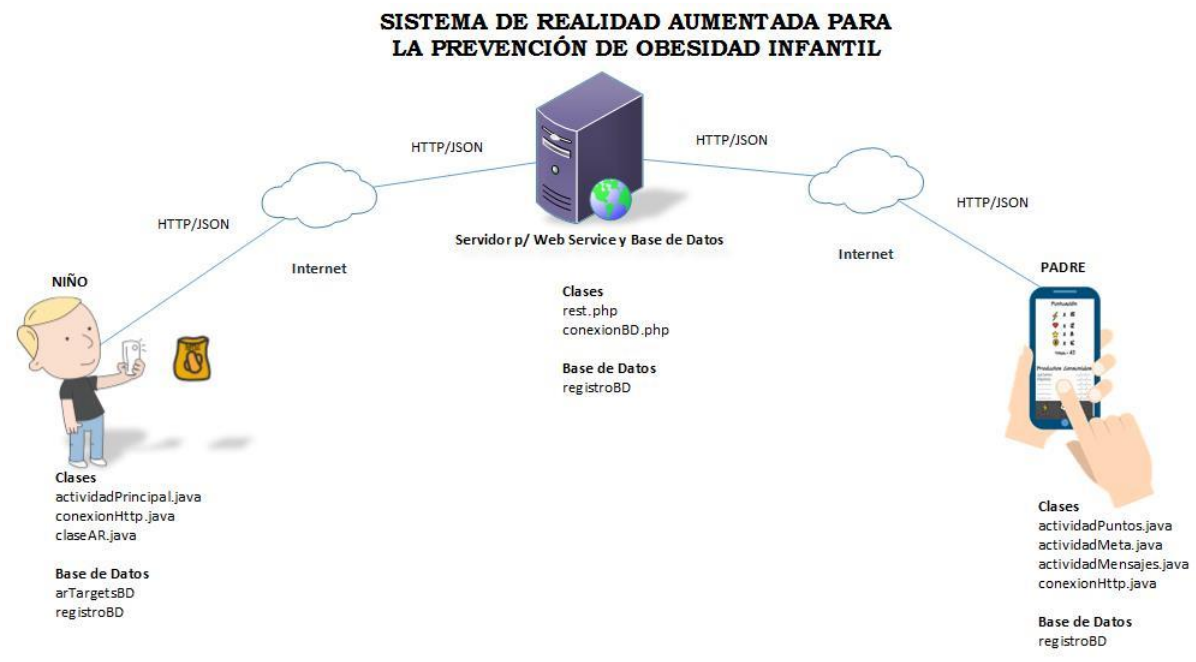

Fig. 3. Estructura del sistema. 


\section{Evaluación de frameworks de RA}

Existen diferentes frameworks de RA para Android en dispositivos móviles. Se seleccionaron algunos para ser probados y ver cuál sería adecuado para utilizarse. Para ello primeramente se definieron criterios de inclusión de frameworks con el fin de evaluar aquellos que puedan y estén al alcance en el futuro desarrollo del sistema, estos criterios están dados de la siguiente forma:

- Acceso total al framework (Incluyendo aquellos con marca de agua).

- Acceso gratuito sin límite de tiempo (Incluyendo aquellos con marca de agua).

- No descontinuado.

- Desarrollo posible en AndroidStudio o Eclipse.

Siguiendo los criterios anteriores los frameworks probados fueron Vuforia, Wikitude y ARToolKit.

Para desarrollar la aplicación de prueba de estos framework se utilizó una computadora con Windows 7, el SDK de cada framework y Eclipse Luna ${ }^{1}$. Para realizar las prueba de la aplicación se usó un SmartPhone Samsung Galaxy s3 sch-i535. En el caso de Vuforia y Wikitude se utilizaron como targets (imágenes a detectar) dos bolsas de golosinas que comúnmente consumen los niños, por otra parte con ARToolKit por su funcionamiento se utilizaron marcadores.

Al utilizar Vuforia se observó el siguiente comportamiento (ver Fig. 4):

- Se detectaron los productos rápidamente.

- Al cambiar la posición y forma (moverla y/o doblarla) de la bolsa la seguía detectando.

- Tarda un poco al cargar la aplicación, pero una vez abierta funciona muy bien.

- Distinción de productos, mostrando para productos diferentes un objeto virtual distinto.

El siguiente framework que se probó fue Wikitude ${ }^{2}$, el funcionamiento observado fue el siguiente (ver Fig. 4):

- Se detectaron los productos rápidamente.

- Es una librería ligera, el tiempo en abrir la cámara es corto.

- Al cambiar la posición y forma del producto (moverla y/o doblarla) normalmente perdía el enfoque y la detección del producto, posteriormente lo vuelve a detectar, oscila este comportamiento.

Posteriormente se evaluó NyARToolkit ${ }^{3}$, el cual se basa en marcadores, para las pruebas se utilizaron dos marcadores distintos (ver Fig. 5).

La detección de los marcadores se hizo correctamente, para cada marcador se generó un objeto distinto, estos solo difieren en su color (ver Fig. 5).

\footnotetext{
${ }^{1}$ https://www.eclipse.org/luna/

${ }^{2} \mathrm{http}: / /$ www.wikitude.com/

${ }^{3}$ http://nyatla.jp/nyartoolkit/wp/
} 

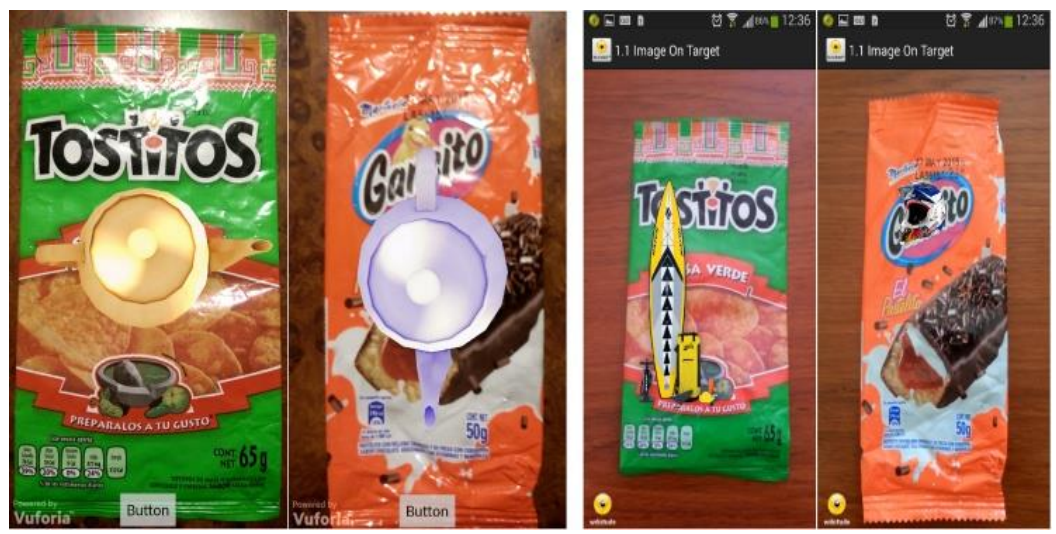

Fig. 4. Identificación de diferentes productos, las primeras dos imágenes son de Vuforia seguido por las de Wikitude.

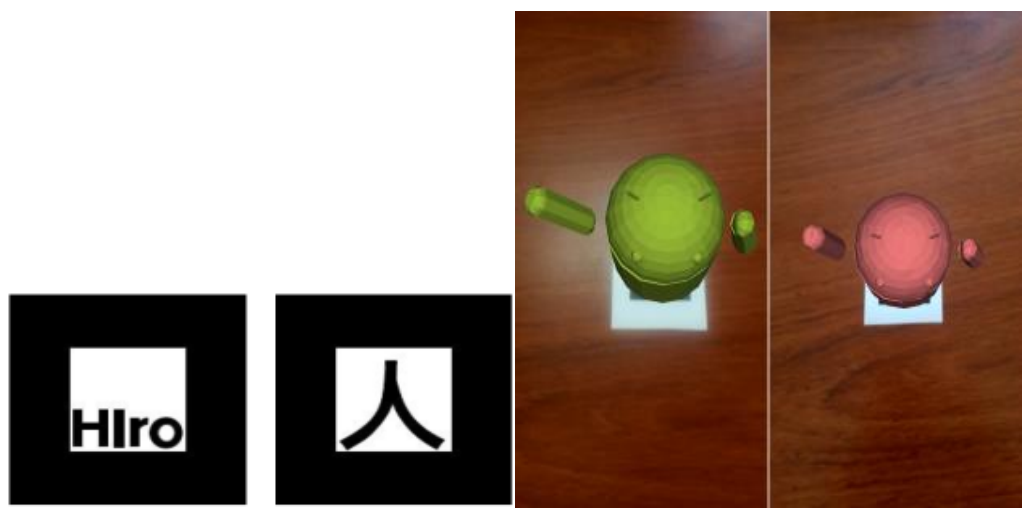

Fig. 5. Identificación de diferentes productos con NyARToolkit.

\section{Resultados}

Como primer resultado se obtuvo el diseño de un sistema interactivo siguiendo Rapid Contextual Design. Este diseño se obtuvo a partir de visioning en el cual se generaron sketches que posteriormente se utilizaron en el storyboarding, en este último se obtuvo un storyboard que se utilizó para diseñar un prototipo. Se trabajó en este prototipo y posteriormente se diseñaron las interfaces gráficas, una para uso del niño y otra para el uso del padre. Este prototipo resultante se pasó a diseño el cual contiene más a detalle la interfaz gráfica de las pantallas. El diseño de la aplicación para niños consta de dos funciones principales, mostrar información nutrimental virtual al identificar un alimento y ver puntaje (Equivalencia entre puntos y valor nutrimental) (ver Fig. 6). Por otra parte, la aplicación para padres consta de tres funciones principales: ver puntos obtenidos por el niño, ver/editar meta (en puntos) y editar mensajes (mensajes alentadores que serán mostrados en la aplicación del niño) (ver Fig. 7). 


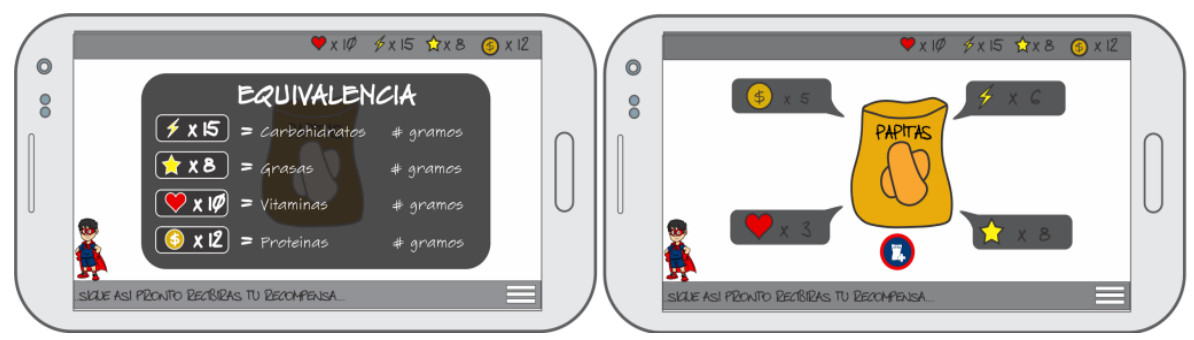

Fig. 6. Pantallas principales de aplicación para el niño ${ }^{4}$.
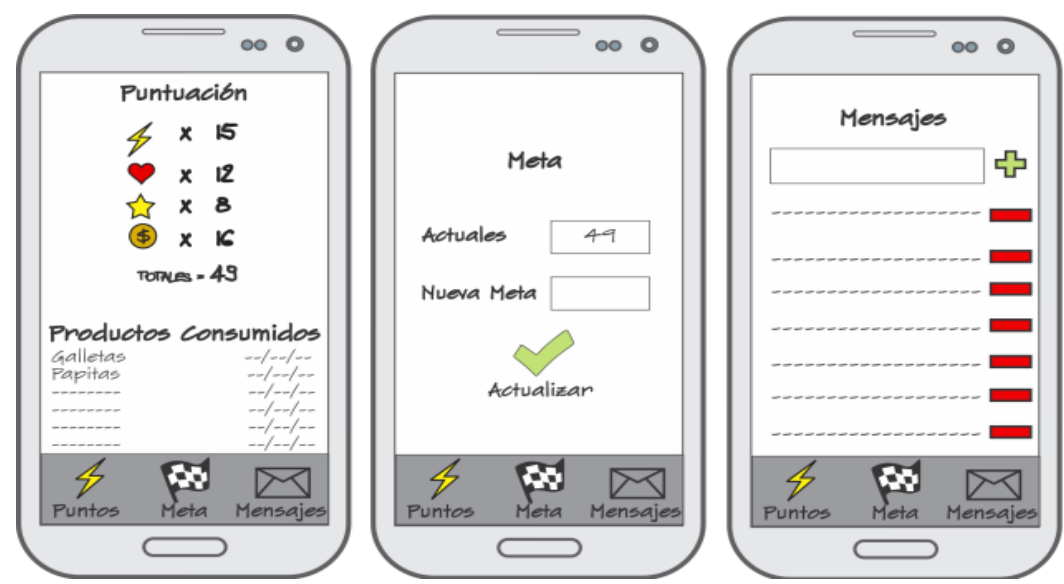

Fig. 7. Pantallas para aplicación del padre (pantalla puntuación, pantalla meta y pantalla mensaje.

Como segundo resultado se obtuvo la evaluación de framework de RA para determinar el adecuado para desarrollar el sistema. Una vez probado cada framework, a partir de una perspectiva propia se obtuvieron los resultados mostrados en la tabla 1. Tomando en cuenta los resultados obtenidos se ve como opción conveniente utilizar Vuforia.

Tabla 1. Resultados de la evaluación de frameworks. La escala utilizada es del uno al cinco donde uno significa "muy malo", dos "malo", tres "regular", cuatro "bueno" y cinco "excelente".

\begin{tabular}{lccc}
\hline & Vuforia & Wikitude & NyArToolKit \\
\hline Rapidez de detección & 5 & 4 & 5 \\
Sencillez de desarrollo & 3 & 4 & 3 \\
Detección al variar la forma del objeto. & 5 & 4 & 3 \\
Velocidad de carga & 4 & 5 & 5 \\
Documentación & 4 & 4 & 3 \\
Tipo de Licencia & Starter* & Trial* & Código Libre \\
\hline
\end{tabular}

${ }^{4}$ Avatar modificado del avatar original disponible en http://galeria.dibujos.net/ con fecha de acceso 14 de abril del 2015.

* Acceso total al framework sin límite de tiempo usando marca de agua. 


\section{Discusión}

En este trabajo se propone una solución tecnológica como auxiliar para prevenir la obesidad infantil en México. La propuesta consiste en un sistema de salud móvil basado en RA para mostrar información nutrimental y orientar a niños en el consumo de alimentos. Se describió el diseño del sistema, el cual se realizó utilizando Rapid Contextual Design. Este diseño permite considerar las perspectivas de los diferentes involucrados que incluye, además de los niños usuarios, a los padres o tutores, profesores de educación básica y médicos especialistas en el área. Como resultado se obtuvo el diseño de un sistema enfocado a niños, considerando principios de diseño ya establecidos y lineamientos encontrados en el análisis de los datos. Por otra parte también se realizó la evaluación de frameworks comerciales existentes para la implementación de sistemas de RA. La evaluación se realizó en consideración a requerimientos propios para el diseño propuesto. Con el fin de extender los resultados de la evaluación a futuras implementaciones, es conveniente seguir realizando pruebas y explorar otras características o funcionalidades de estos mismos frameworks, así como detectar limitaciones en cuanto a los requerimientos de los sistemas de este tipo.

\section{Referencias}

1. UNICEF México, Salud y Nutrición, http://www.unicef.org/mexico/spanish/17047.htm

2. Encuesta Nacional de Salud y Nutrición Resultados Nacionales 2012, http://ensanut.insp.mx/informes/ENSANUT2012ResultadosNacionales.pdf

3. OMS, Estrategia mundial sobre régimen alimentario, actividad física y salud, http://www.who.int/dietphysicalactivity/childhood_why/es/

4. Billinghurst, M.: Augmented reality in education. New Horizons for Learning, 12 (2002)

5. Daponte, P., De Vito, L., Picariello, F., Riccio, M.: State of the art and future developments of the Augmented Reality for measurement applications. Measurement, 57, pp. 53-70 (2014)

6. Rogers, Y., Sharp, H., Preece, J.: Interaction design: beyond human-computer interaction. John Wiley \& Sons (2011)

7. Santos, M.E.C., Chen, A., Taketomi, T., Yamamoto, G., Miyazaki, J., Kato, H.: Augmented reality learning experiences: Survey of prototype design and evaluation. Learning Technologies, IEEE Transactions on, Vol. 7, No. 1, pp. 38-56 (2014)

8. Bayu, M.Z., Arshad, H., Ali, N.M.: Nutritional Information Visualization Using Mobile Augmented Reality Technology. Procedia Technology, 11, pp. 396-402 (2013)

9. Philips, Vital Signs Camera, http://www.vitalsignscamera.com/how-it-works.html 